\title{
Stathmin expression in glioma-derived microvascular endothelial cells: A novel therapeutic target
}

\author{
BAIJING DONG $^{1 *}$, LUYAN MU $^{1 *}$, XIANGYING QIN ${ }^{1}$, WANCHEN QIAO ${ }^{1}$, \\ XIAODONG LIU ${ }^{2}$, LIMING YANG ${ }^{3}$, LI XUE ${ }^{4}$, NIKOLAI G. RAINOV ${ }^{5}$ and XIAOQIAN LIU ${ }^{1}$ \\ ${ }^{1}$ Department of Neurosurgery, the Fourth Affiliated Hospital of Harbin Medical University, Harbin 150001; \\ ${ }^{2}$ Department of Neurosurgery, Tonghua Municipal People's Hospital, Tonghua 134001; ${ }^{3}$ Department of Pathophysiology, \\ Harbin Medical University; ${ }^{4}$ Department of Clinical Laboratory, the Fourth Affiliated Hospital of Harbin Medical University, \\ Harbin 150001, P.R. China; ${ }^{5}$ Department of Neurosurgery, Klinikum Augsburg, D-86156 Augsburg, Germany
}

Received September 13, 2011; Accepted October 14, 2011

DOI: $10.3892 /$ or.2011.1525

\begin{abstract}
The purpose of this study was to investigate stathmin expression and its mechanisms of action in GDMEC. Microvascular endothelial cells were isolated from human gliomas $(n=68)$ and normal brain specimans $(n=20)$, and purified by magnetic beads coated with anti-CD105 antibody. The expression of stathmin mRNA and protein were detected by RT-PCR and western blotting, respectively. Stathmin expression was silenced by application of specific siRNA in high grade GDMEC. The proliferation, apoptosis and invasion behavior of GDMEC were investigated. The stathmin positive rate of endothelial cells in normal brain, grade I-II glioma and grade III-IV glioma was 20,66 and 95.5\%, respectively $(\mathrm{P}<0.05)$. When cells were treated with siRNA to silence stathmin, cell viability was reduced, the apoptosis rate increased and the migration of vascular endothelial cells was suppressed significantly $(\mathrm{P}<0.05)$. Down-regulation of stathmin suppressed neoangiogenesis of glioma and provides a potential target for glioma treatment.
\end{abstract}

\section{Introduction}

Glioma is the most common and aggressive form of primary brain tumors. High grade gliomas (anaplastic glioma/AA, WHO grade III, and glioblastoma multiforme/GBM, WHO grade IV) displays unique biological features especially network of neoplastic blood vessels, invasion and metastasis. The current standard treatment schemes for high grade glioma include gross total resection, radiotherapy, and concomitant and/or sequential

Correspondence to: Dr Xiaoqian Liu, Department of Neurosurgery, the Fourth Affiliated Hospital of Harbin Medical University, 37th of Yiyuan Street, Harbin 150001, P.R. China

E-mail: liuxiaoqian@vip.163.com

*Contributed equally

Key words: vascular endothelial cells, glioma, neoangiogenesis, stathmin oral chemotherapy with temozolomide. Although these treatments may significantly prolong survival, they are not curative.

With the development of molecular biology and RNAi techniques, the molecular genetic markers and chemotherapy resistance mechanisms of glioma have been extensively investigated. In 1971, Folkman (1) proposed that tumor growth and metastasis depend on angiogenesis, and that inhibition of angiogenesis may be an effective strategy for cancer treatment. Since microvasular endothelial cell is the key player in the process of neoangiogenesis, molecular genetic investigations of microvasular endothelial cells have been extensively carried out. Most of the researches have been concentrated on VEGF and its analogs within the VEGF receptor-mediated signaling cascades $(2,3)$.

Stathmin (synonymous with oncoprotein 18/OP18), also known as metablastin, is a member of the microtubule destabilizing protein family. It regulates microtubule dynamics during cell cycle progress $(4,5)$. The activity of stathmin is regulated by phosphorylation during the cellular transition from interphase to metaphase. The non-phosphorylated stathmin promotes the depolymerization of microtubules by sequestering tubulin (6) while the phosphorylated stathmin leads to increasing microtubule stabilization which promotes formation of a mitotic spindle (7).

Stathmin is overexpressed in various human malignancies, such as leukemia, neuroblastoma and mesothelioma (8). Most studies have demonstrated that expression of stathmin is associated with tumor progression and unfavorable long-term prognosis. However, the expression of stathmin in human GDMEC (glioma-derived microvascular endothelial cells) and NDMEC (normal brain tissue-derived microvascular endothelial cells) has not been previously investigated.

Recent studies have revealed significant differences in stathmin between VEC (vascular endothelial cells) lines and newly isolated tissue-specific microvasular endothelial cell (9-12). However, there were several studies of NDMEC and GDMEC, which showed that GDMEC have stronger ability of angiogenesis and invasion $(13,14)$. We have developed a reliable and reproducible method for microvascular endothelial cell isolation from human glioma of different histological grade and have examined the expression of stathmin in human 
GDMEC and NDMEC. In addition, we evaluated the effect of siRNA-mediated silencing of the stathmin gene.

\section{Materials and methods}

Tissue collection. Glioma samples were obtained from 68 patients (30 male, 38 female; age range: 13-74 years) who had surgical resection of glioma between 2008 and 2010 . Normal brain tissue were obtained from 20 patients (12 male, 8 female; age range: 20-45 years) who underwent decompressive surgical procedures for severe head injury within the same time period. The protocol for this study was approved by the local Ethics Committee of our Hospital. Gliomas were of different histological grades: 10 pilocytic astrocytomas (WHO I), 14 low-grade astrocytomas (WHO II), 24 anaplastic astrocytomas (WHO III), and 20 glioblastomas (WHO IV).

Cell isolation and culture. Fresh tumor tissue was washed with PBS containing penicillin-streptomycin and then minced into pieces $\sim 1 \mathrm{~mm}^{3}$. Following digestion with $0.25 \%$ trypsin (Sigma-Aldrich) at $37^{\circ} \mathrm{C}$ for $30 \mathrm{~min}$, further digested by $1 \mathrm{mg} /$ $\mathrm{ml}$ collagenase (Invitrogen) at $37^{\circ} \mathrm{C}$ for $1 \mathrm{~h}$, and the mixture was filtered with sterile steel mesh. After centrifugation at $1000 \mathrm{rpm}$ for $5 \mathrm{~min}$, the cells were suspended with complete DMEM medium, then placed in $2 \%$ gelatin-coated tissue culture flasks at $37^{\circ} \mathrm{C}$ for 7 days to yield an enriched cell population. Cells were purified by incubating with magnetic MACS (Miltenyi Biotec, Germany) coated with a monoclonal anti-CD105 antibody according to the manufacturer's instructions (15). GDMEC and NDMEC of second passage were used for study. Characterization of microvasular endothelial cells was performed by FVIII-Rag stain with cellular immunity (Fig. 1A).

Immunohistochemistry. One part of each tissue was fixed with formalin and then embedded with paraffin, and sliced into $4-\mu \mathrm{m}$ sections. Following deparaffinization and hydration, the slides were treated with $0.3 \%$ peroxide for $15 \mathrm{~min}$, after which the sections were blocked with $1.5 \%$ blocking serum (Invitrogen) for $2 \mathrm{~h}$ at room temperature. A rabbit anti-human stathmin polyclonal primary antibody (CST, USA) was applied in overnight incubation. After washing with PBS thrice and incubating with goat anti-rabbit secondary antibody at $37^{\circ} \mathrm{C}$ for $30 \mathrm{~min}$, the slides were treated with DAB (ZhongShan, Co. Ltd., Beijing, China). Finally, the slides were lightly counterstained with hematoxylin, dehydrated and mounted. The mean percentage of stathmin-positive cells of each sample was determined by counting at least 5 random visual fields with microscope at $\mathrm{x} 400$ magnification.

RNA interference. High grade GDMEC at $10^{5}$ cells $/ \mathrm{ml}$ were transfected in duplicate with $80 \mathrm{nM}$ siRNA pools (2 siRNA duplexes) targeting the stathmin. Lipofectamine 2000 and siRNA were diluted in $50 \mu \mathrm{l}$ Opti-MEM I at a ratio of 1:25 and incubated at room temperature for $5 \mathrm{~min}$, respectively. Equal volume of the siRNA and Lipofectamine 2000 solutions were mixed and incubated at room temperature for another $15 \mathrm{~min}$ with gentle shaking, then the mixture was added into the cell culture. After overnight incubation, the cells were cultured in complete medium for 2 days.
Cell proliferation assays. High grade GDMEC were plated in 96-well plates (6,000/well) and transfected as mentioned above. Transfected and non-transfected high grade GDMEC were incubated for 24,48 , or $72 \mathrm{~h}$, respectively. Then cell proliferation was analyzed by MTT colorimetric assay. The cells were incubated with MTT solution (1 mg/ml in RPMI-1640) for $4 \mathrm{~h}$ at $37^{\circ} \mathrm{C}$, then medium was decanted, and $150 \mu 1 \mathrm{DMSO}$ was added into each well. Absorbance was measured at $570 \mathrm{~nm}$ using an ELISA Reader (RT-2100C, Rayto, USA). Experiments were performed in triplicate.

Cell apoptosis assay. After designated treatment, cells were washed, harvested, and counted, then cells $\left(1 \times 10^{5}\right)$ were re-suspended in $100 \mu \mathrm{l}$ binding buffer, before $10 \mu \mathrm{l}$ of Annexin V and $5 \mu 1$ of PI were added, and incubated in the dark for $15 \mathrm{~min}$ at room temperature, according to the manufacturer's instructions (Biosea, China). The apoptosis rate (\%) was determined with a cytometer (Epics Altra II, Beckman Coulter, USA). Cells were also viewed under an inverse fluorescent microscope. The experiments were repeated thrice.

Western blotting. Total protein was extracted and then electrophoresis was performed by SDS-PAGE and transferred onto PVDF membrane (Millipore, Bedford, MA, USA), followed by blocking with 5\% skimmed milk dissolved in TBS contained $1 \%$ Tween-20 for $1 \mathrm{~h}$ at room temperature. The membrane was incubated with primary antibody at $4^{\circ} \mathrm{C}$ overnight, and incubated in alkaline phosphatase-conjugated secondary antibody for $1 \mathrm{~h}$ at room temperature after washing with TBST three times. After washing, the bands of protein were detected with NBT/BCIP substrates (ZhongShan, Co. Ltd). The specific antibodies were anti-stathmin rabbit polyclonal antibody (Cell Signal Tech), rabbit polyclonal Bcl-X antibody, rabbit polyclonal Bcl-2 antibody, rabbit polyclonal Bax antibody, and rabbit polyclonal pro-Caspase-3 antibody (Santa-Cruz Biotech).

Reverse transcription PCR. Total RNA was extracted with the TRIzol reagent (Invitrogen) following the manufacturer's protocol. Total RNA was retro-transcripted using MMLV reverse transcriptase. Oligo(d)T (Promega, USA) including $2 \mu \mathrm{g}$ RNA of the obtained cDNA was then amplified using primers (SanGon, Shanghai, China) for the human stathmin and GAPDH sequences. The sequences and the reaction conditions are described in Table I. PCR products were separated on $2 \%$ agarose gel and quantified by densitometry.

Invasion assay. Appropriate Matrigel (BD Biosciences, Bedford, MA, USA) was added to the upper chamber of the transwell apparatus with $8-\mu \mathrm{m}$ pore size membrane (Costar, Cambridge, MA, USA). After the Matrigel solidified at $37^{\circ} \mathrm{C}$, serum-free DMEM containing $1 \times 10^{5}$ siRNA-transfected and control high grade GDMEC in $100 \mu \mathrm{l}$ were added into the upper chamber. The lower chamber was loaded with $500 \mu 1$ medium with $10 \%$ FBS. After incubation at $37^{\circ} \mathrm{C}$ for $24 \mathrm{~h}$, membranes coated with matrigel were wiped with a cotton swab and fixed with $100 \%$ methanol for $10 \mathrm{~min}$. The membranes with cells were soaked in $0.1 \%$ crystal violet for $10 \mathrm{~min}$ and then washed with distilled water. The number of cells attached to the lower surface of the polycarbonate filter was counted at $\times 400$ magnification 
Table I. The sequences of primer for RT-PCR. ${ }^{a}$

\begin{tabular}{lll}
\hline Gene & \multicolumn{1}{c}{ Sequences } & \multicolumn{1}{c}{ Procedure } \\
\hline Stathmin & F: 5'-ACTGCCTGTCGCTTGTCT-3' & $95^{\circ} \mathrm{C}$ for 3 min \\
& R: 5'-GTCTCGTCAGCAGGGTCT-3' & $95^{\circ} \mathrm{C}$ for $30 \mathrm{sec}, 57^{\circ} \mathrm{C}$ for $30 \mathrm{sec}, 72^{\circ} \mathrm{C}$ for $40 \mathrm{sec}$, \\
GAPDH & F: 5'-GGTGAAGGTCGGAGTCAACGG-3' & 30 cycles; \\
& R: 5'-CCTGGAAGATGGTGATGGGATT-3' & Final elongation step at $72^{\circ} \mathrm{C}, 10 \mathrm{~min}$.
\end{tabular}

${ }^{\mathrm{a}}$ All sequences were designed with PrimerPremier 5.0.
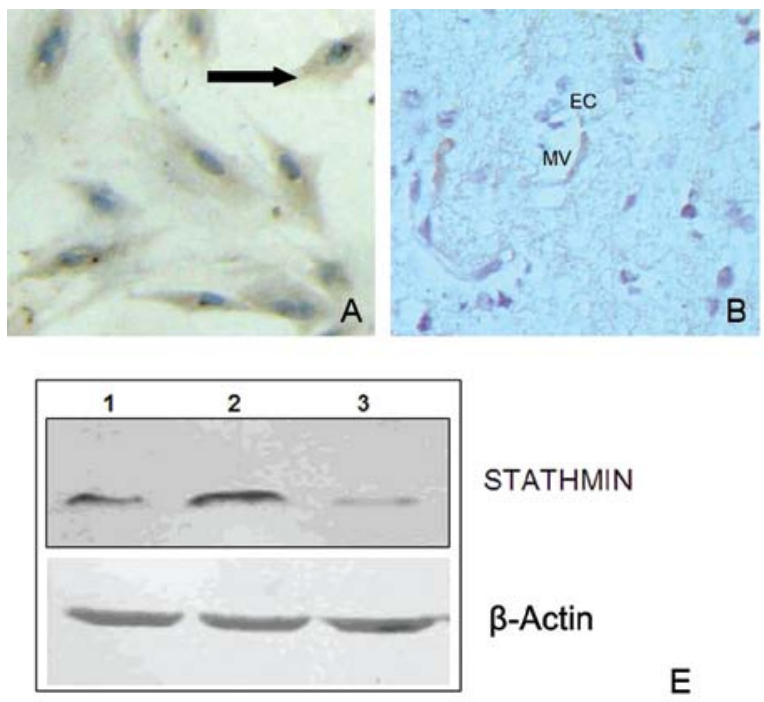

STATHMIN

B-Actin
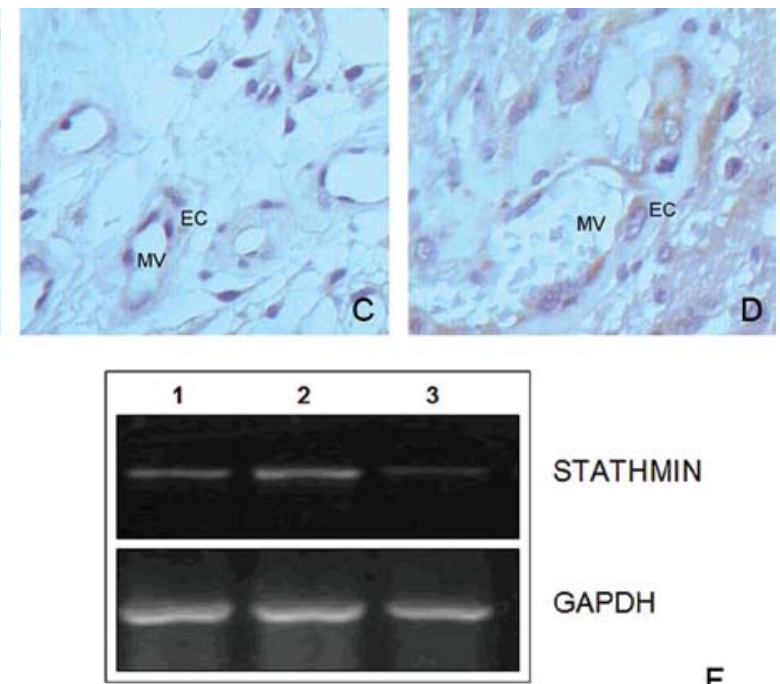

STATHMIN

GAPDH

Figure 1. Immunohistochemical staining for FVIII-Rag in cultured GDMEC (DAB staining appears brown-colored; original magnification, $\mathrm{x} 400$ ). (B-D) Analysis of stathmin expression in human glioma by immunohistochemistry. Stathmin was detected mainly in the cytoplasm of VEC from microvessels and in glial cells (original magnification, $x 400$; counterstaining with H\&E). MV, microvessel; EC, endothelial cell. (B) Expression of stathmin in normal brain. (C) Expression of stathmin in low-grade glioma (WHO grade I-II). (D) Expression of stathmin in high grade glioma (WHO grade III-IV). (E) Western blot analysis of stathmin protein expression in GDMEC and NDMEC, lanes: 1, GDMEC from WHO I-II tumors; 2, GDMEC from grade III-IV tumors; 3, NDMEC. (F) RT-PCR analysis of stathmin mRNA in GDMEC and NDMEC, lanes: 1, GDMEC from WHO I-II tumors; 2, GDMEC from WHO III-IV tumors; 3, NDMEC.

Table II. The statistical results of immunohistochemistry. ${ }^{a}$

\begin{tabular}{lccc}
\hline Group & Stathmin (-) & Stathmin (+) & P-value \\
\hline Normal Brain & 16 & 4 & $<0.001$ \\
Glioma & 10 & 58 & \\
Glioma & & & \\
Grade I-II & 8 & 16 & $<0.05$ \\
Grade III-IV & 2 & 42 &
\end{tabular}

${ }^{\text {aT }}$ The tissue was regarded as negative when the positive cell rate was $<10 \%$.

under a light microscope. Results were expressed as the mean of triplicate experiments.

Statistical analysis. Statistical analysis was performed using SPSS 16.0 statistical software (SPSS, Chicago, IL), differences between groups were analyzed by $\chi^{2}$ test or t-test and $\mathrm{P}<0.05$ was considered as statistically significant.

\section{Results}

Stathmin expression in VEC is associated with histological grade of glioma. Immunohistochemical analysis demonstrated that the percentages of stathmin-positive VEC in normal human brain tissue, WHO grade I-II glioma, and WHO grade III-IV glioma were 20, 66 and $95.5 \%$, respectively. The numbers of stathmin-positive VEC increased with increasing histological grade of tumor (Fig. 1B-D, Table II). The expression of stathmin protein and transcription of stathmin mRNA were significantly increased in GDMEC from high-grade glioma compared with GDMEC from low grade glioma and NDMEC (Fig. 1E and F).

Stathmin silencing may suppress proliferation and induce apoptosis of GDMEC. Application of siRNA to silence stathmin in GDMEC resulted in reduced expression of stathmin protein as well as inhibited mRNA transcription (Fig. 2A and B). Cell proliferation was significantly suppressed 

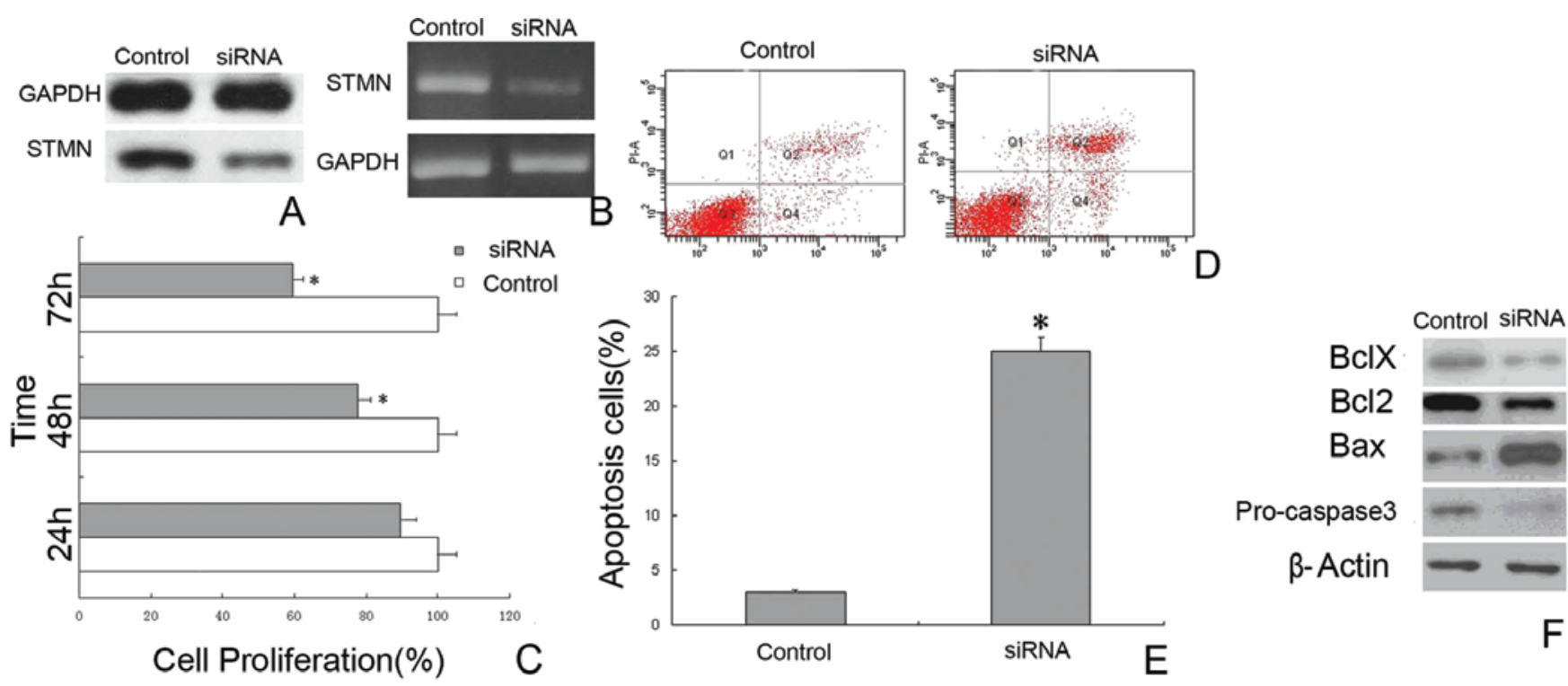

Figure 2. (A) Western blot analysis on stathmin expression of siRNA transfection. (B) RT-PCR analysis on stathmin transcription of siRNA transfection. (C) Proliferation of GDMEC in the siRNA group is suppressed, the difference between the siRNA and control groups is statistically significant ("P<0.05). (D and E) The apoptosis rate of siRNA-transfected GDMEC is significantly higher than the control group ( $\left.{ }^{*} \mathrm{P}<0.05\right)$. (F) Western blot demonstrates that expression of apoptosis associated proteins. Bcl-X, Bcl-2 and pro-caspase-3 were down-regulated while Bax was up-regulated in the siRNA-transfected group.

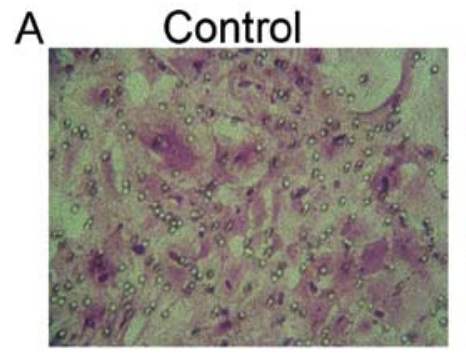

B

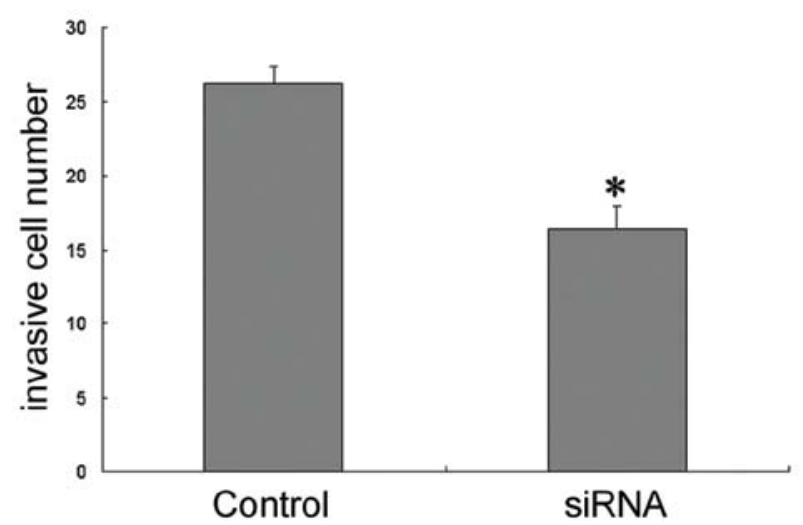

Figure 3. (A) Matrigel transwell assays for analysis of GDMEC invasion (original magnification, $x 400$ ). (B) Graph shows invading cell numbers in the siRNA and the control groups. The difference between groups is statistically significant $\left({ }^{*} \mathrm{P}<0.05\right)$.

(Fig. 2C) and the rate of apoptosis was increased (Fig. 2D and E). Proteins inducing apoptosis were up-regulated while anti-apoptosis proteins were down-regulated (Fig. 2F). These results demonstrated that siRNA-mediated silencing of stathmin could suppress proliferation and induce apoptosis in GDMEC.
siRNA mediated silence of stathmin may suppress the migratory ability of GDMEC. The results of Matrigel Transwell analysis showed that the invasive capacity of GDMEC derived from high grade glioma was significantly reduced by siRNA transfection and stathmin silencing (Fig. 3). Cell number of siRNA group passing through the matrigel was significantly lower than the control group. The results demonstrated that siRNA-mediated stathmin silence suppressed metastasis of GDMEC and inhibited invasion of gliomas.

\section{Discussion}

Angiogenesis is a crucial step in solid tumor growth and metastasis (16). Currently it is agreed that the growth of tumors can be divided into two stages (17). Stage I indicates the slow non-vascular growth of small cell clusters without neovascularisation, and stage II indicates the angiogenesis phase when tumor blood vessels could provide nutrition for a larger cell cluster. In stage II, the tumor grows rapidly and scattered cells may infiltrate surrounding tissue and metastasize along neocapillaries. Angiogenesis provides not only the prerequisite for the survival but also a route for tumor cell invasion and metastasis. Microvascular endothelial cell is an indispensable component of the vascular system and plays a pivotal role in the above described processes.

The cytoskeleton experiences dramatic structural rearrangements during the various stages of angiogenesis, including proliferation, migration, and differentiation of microvascular endothelial cells into vascular structures $(18,19)$. Stathmin is an important protein that regulates cellular microtubule dynamics, and promotes mitotic spindle formation during mitosis (20-23). A potential role for stathmin in angiogenesis is that stathmin expression may be regulated by vascular endothelial zinc finger 1 (Vezf1). When antisense oligonucleotides targeted 
Vezf1 were applied, the inhibition of stathmin expression was observed (24)

A recent study by Mistry et al shows that application of anti-stathmin ribozyme to human umbilical vascular microvasular endothelial cells results in inhibition of angiogenesis (25). In this study, we confirmed that the level of stathmin expression in GDMEC was significantly higher than NDMEC. Immunohistochemical analysis showed that glioma-derived microvasular endothelial cells exhibited moderate to strong stathmin staining (mainly in the cytoplasm), whereas stathmin staining was weak in microvasular endothelial cells from normal brain. Further, we found that the stathmin expression was significantly higher in cultured GDMEC compared with NDMEC at both transcriptional and translational level. The expression of stathmin in VEC was intimately associated with the WHO grade.

As WHO grade III-IV gliomas have a locally aggressive behavior and the VEC from this grade strongly express stathmin, we selected high grade GDMEC for the next investigation. The transcription and expression of stathmin was suppressed by transfection of cells with specific siRNA, which was also able to reduce the proliferation rate and the invasive potential of GDMEC. All these findings indicate that stathmin might play an important role in glioma neoangiogenesis and may exert its action by different mechanisms. Also, stathmin is a potential target of neoangiogenesis suppression in glioma treatment.

Further elucidation of the mechanisms of tumor angiogenesis may provide more precise and effective anticancer therapies. Comparative studies of tumor-derived microvasular endothelial cell and microvascular endothelial cells from nonneoplastic tissues seem to be best suited for such differential expression studies. Our findings suggested that stathmin plays an important role in glioma progression by supporting neoangiogenesis. Stathmin is a promising therapeutic target molecule in human glioma.

\section{Acknowledgements}

This study was supported by grant no. 201023 from the Office of Human Resources and Social Security of Heilongjiang Province, China. The authors would like to thank Mr. Yuwen Song and Ms. Quanye Sun for technic and experimental support.

\section{References}

1. Folkman J: Tumor angiogenesis: therapeutic implications. N Engl J Med 285: 1182-1186, 1971.

2. Sato K, Tsuchiya N, Sasaki R, et al: Increased serum levels of vascular endothelial growth factor in patients with renal cell carcinoma. J Cancer Res 90: 874-879, 1999.

3. Yagasaki H, Kawata N, Takimoto Y, et al: Histopathological analysis of angiogenic factors in renal cell carcinoma. Int J Urol 10: 220-227, 2003 .

4. Rubin CI and Atweh GF: The role of stathmin in the regulation of the cell cycle. J Cell Biochem 93: 242-250, 2004.
5. Bhat KM and Setaluri V: Microtubule-associated proteins as targets in cancer chemotherapy. Clin Cancer Res 13: 2849-2854, 2007.

6. Leibl S, Zigeuner R, Hutterer G, et al: EGFR expression in urothelial carcinoma of the upper urinary tract is associated with disease progression and metaplastic morphology. APMIS 116: 27-32, 2008.

7. Cassimeris L: The oncoprotein 18/stathmin family of microtubule destabilizers. Curr Opin Cell Biol 14: 18-24, 2002.

8. Rana S, Maples PB, Senzer N, et al: Stathmin 1: a novel therapeutic target for anticancer activity. Expert Rev Anticancer Ther 8: 1461-1470, 2008.

9. Nanda A and St Croix B: Tumor endothelial markers: new targets for cancer therapy. Curr Opin Oncol 16: 44-49, 2004.

10. St Croix B, Rago C, Velculescu V, et al: Genes expressed in human tumor endothelium. Science 289: 1197-1202, 2000.

11. Bussolati B, Deambrosis I, Russo S, et al: Altered angiogenesis and survival in human tumor-derived endothelial cells. FASEB J 17: 1159-1161, 2003.

12. Zhang X, Yamashita M, Uetsuki H, et al: Angiogenesis in renal cell carcinoma: evaluation of microvessel density, vascular endothelial growth factor and matrix metalloproteinases. Int J Urol 9: 509-514, 2002

13. Unger RE, Oltrogge JB, Von Briesen $\mathrm{H}$, et al: Isolation and molecular characterization of brain microvascular endothelial cells from human brain tumors. In Vitro Cell Dev Biol Anim 38: 273-281, 2002.

14. Bian XW, Jiang XF, Chen JH, et al: Increased angiogenic capabilities of endothelial cells from microvessels of malignant human gliomas. Int Immunopharmacol 6: 90-99, 2006.

15. Miebach S, Grau S, Hummel V, et al: Isolation and culture of microvascular endothelial cells from gliomas of different WHO Grades. J Neurooncol 76: 39-48, 2006.

16. She X, Matsuno F, Harada N, et al: Synergy between antiendoglin (CD105) monoclonal antibodies and TGF-beta in suppression of growth of human endothelial cells. Int J Cancer 108: 251-257, 2004.

17. Naumov GN, Akslen LA and Folkman J: Role of angiogenesis in human tumor dormancy: animal models of the angiogenic switch. Cell Cycle 5: 1779-1787, 2006.

18. Ingber D, Prusty D, Zhengqi S, et al: Cell shape, cytoskeletal mechanics and cell cycle control in angiogenesis. J Biomech 28: 1471-1484, 1995

19. Hayot C, Farinelle S, De Decker R, et al: In vitro pharmacological characterizations of the anti-angiogenic and anti-tumor cell migration properties mediated by microtubule-affecting drugs, with special emphasis on the organization of the actin cytoskeleton. Int J Oncol 21: 417-425, 2002.

20. Holmfeldt P, Sellin ME and Gullberg M: Predominant regulators of tubulin monomer-polymer partitioning and their implication for cell polarization. Cell Mol Life Sci 66: 3263-3276, 2009.

21. Sellin ME, Holmfeldt P, Stenmark $S$, et al: Global regulation of the interphase microtubule system by abundantly expressed Op18/stathmin. Mol Biol Cell 19: 2897-2906, 2008.

22. Budhachandra K, Brojen Singh RK and Menon GI: Microtubule dynamics regulated by stathmin. Comput Biol Chem 32: 141-144, 2008.

23. Mistry SJ and Atweh GF: Role of stathmin in the regulation of the mitotic spindle: Potential applications in cancer therapy. Mt Sinai J Med 69: 299-304, 2002

24. Miyashita H, Kanemura M, Abe M, et al: Vascular endothelial zinc finger1 is i nvolved in the regulation of angiogenesis: possible contribution of stathmin/Op18 as a downstream target gene. Arterioscler Thromb Vasc Biol 24: 878-884, 2004.

25. Mistry SJ, Bank A and Atweh GF: Synergistic antiangiogenic effects of stathmin inhibition and taxol exposure. Mol Cancer Res 5: 773-782, 2007. 